\title{
Regulation of Apoptosis by HER2 in Breast Cancer
}

\section{Richard L Carpenter ${ }^{1}$ and Hui-Wen Lo ${ }^{1,2 *}$}

${ }^{1}$ Division of Surgical Sciences, Department of Surgery, Duke University School of Medicine, Durham, North Carolina 27710, USA

${ }^{2}$ Duke Cancer Institute, Duke University School of Medicine, Durham, North Carolina 27710, USA

\begin{abstract}
HER2 is a trans-membrane receptor tyrosine kinase that activates multiple growth-promoting signaling pathways including PI3K-AKT and Ras-MAPK. Dysregulation of HER2 is a frequent occurrence in breast cancer that is associated with poor patient outcomes. A primary function of HER2 is suppressing apoptosis to enhance cell survival giving rise to uncontrolled proliferation and tumor growth. There has been much investigation into the mechanisms by which apoptosis is suppressed by HER2 in hopes of finding clinical targets for HER2-positive breast cancers as these cancers often become resistant to therapies that directly target HER2. Several apoptotic mechanisms have been shown to be deregulated in HER2-overexpressing cells with examples in both the intrinsic and extrinsic apoptotic pathways. HER2-mediated activation of PI3K-AKT signaling is required for many of the mechanisms HER2 uses to suppress apoptosis. HER2 overexpression is correlated with increases in anti-apoptotic Bcl-2 proteins including $\mathrm{Bcl}-2, \mathrm{Bcl}-\mathrm{xL}$, and $\mathrm{Mcl}-1$. HER2 also suppresses p53-mediated apoptosis by upregulation of MDM2 by activation of AKT. In addition, survivin expression is often increased with HER2 overexpression leading to inhibition of caspase activation. There is also recent evidence to suggest HER2 can directly influence apoptosis by translocation to the mitochondria to inhibit cytochrome $c$ release. HER2 can also suppress cellular reaction to death ligands, especially TRAIL-induced apoptosis. Elucidation of the mechanisms of apoptotic suppression by HER2 suggest that clinical treatment will likely need to target multiple components of these pathways as there is redundancy in HER2-mediated cell survival. Several therapies have attempted to target Bcl-2 proteins that have promising pre-clinical results. Next-generation HER2 targeting therapies include irreversible pan-ERBB inhibitors and antibody-drug conjugates, such as T-DM1 that has very promising clinical results thus far. Further investigation should include elucidating mechanisms of resistance to HER2-targeted therapies and targeting of multiple components of HER2-mediated cell survival.
\end{abstract}

Keywords: HER2; ERBB; Bcl-2; Apoptosis; AKT; p53; Survivin

\section{Introduction}

HER2 (or ERBB2/Neu) is a transmembrane tyrosine kinase belonging to the ERBB family of receptors (ERBB1-4). ERBB receptors hetero and homo-dimerize resulting in activation of tyrosine kinase activity. ERBB tyrosine receptor kinases activate several pathways, including PI3K-AKT and RAS-MAPK pathways, which regulate many cell functions including proliferation, migration, survival, and cell growth among others. Hyperactivation or overexpression of these receptors can lead to uncontrolled cell growth and proliferation leading to cancer development. HER2, specifically, is overexpressed in 20$30 \%$ of breast cancers and this overexpression is associated with poor patient outcomes [1-3]. One of the hallmarks of cancer is the resistance to apoptosis [4,5] and HER2 overexpression leads to suppression of apoptosis. This review will focus on the known mechanisms by which HER2 suppresses apoptosis in breast cancer cells.

\section{Mechanisms of Apoptosis}

Apoptosis, or programmed cell death, occurs in human cells via two primary pathways termed the intrinsic and extrinsic pathways. The intrinsic pathway is mediated by the mitochondria whereby the outer mitochondrial membrane loses its integrity in a process termed outer Mitochondrial Membrane Permeabilization (MOMP), which allows the release of pro-apoptotic proteins. Cytochrome $c$, one of the released pro-apoptotic proteins, then interacts with apoptotic protease-activating factor 1 (APAF1). APAF1 oligomerizes forming the apoptosome, which recruits and activates caspase 9, an initiator caspase. MOMP also causes release of Second Mitochondria-Derived Activator of Caspase (SMAC or DIABLO) and OMI (HTRA2). These proteins indirectly enhance caspase activity by preventing caspase inhibition by inhibitor of apoptosis proteins (IAPs). IAPs, including
$\mathrm{XIAP}$ and survivin, play a significant role in suppression of apoptosis in cancer cells.

The balance of pro- and anti-apoptotic proteins in the $\mathrm{Bcl}-2$ protein family primarily regulates the intrinsic pathway and MOMP. The Bcl2 proteins Bax and Bak mediate initiation of MOMP. MOMP begins with Bax localization to the mitochondrial outer membrane (MOM) where it interacts with Bak leading to pore formation and release of cytochrome $c$. Anti-apoptotic Bcl-2 proteins (Bcl-2, Bcl-xL, Mcl-1, $\mathrm{Bcl}-\mathrm{w}$, and A1) can physically interact with Bax/Bak suppressing their pore-forming function. Another category of $\mathrm{Bcl}-2$ proteins are the proapoptotic BH3-only proteins that include PUMA, NOXA, Bik, Bim, and $\mathrm{Bad}$, among others. These $\mathrm{BH} 3$-only proteins promote apoptosis by two mechanisms: 1) physical interaction with anti-apoptotic Bcl-2 proteins preventing their inhibition of $\mathrm{Bax} / \mathrm{Bak}$ and 2) direct activation of Bax/Bak. The balance between pro-apoptotic and anti-apoptotic $\mathrm{Bcl}-2$ proteins plays a large role in whether MOMP, and ultimately apoptosis, will occur. P53 also participates in promoting apoptosis by sensing DNA damage and can upregulate pro-apoptotic Bcl-2 proteins (e.g. PUMA) as well as suppress IAPs (e.g. survivin).

The extrinsic apoptotic pathway is directly activated by external

*Corresponding author: Hui-Wen Lo, Division of Surgical Sciences Department of Surgery (Box 3156), Duke University School of Medicine, Duke Cancer Institute, 423 MSRB I, 103 Research Drive, Durham, NC 27710, USA, E-mail: huiwen.lo@duke.edu

Received April 20, 2013; Accepted June 17, 2013; Published June 26, 2013

Citation: Carpenter RL, Lo HW (2013) Regulation of Apoptosis by HER2 in Breast Cancer. J Carcinog Mutagen S7: 003. doi:10.4172/2157-2518.S7-003

Copyright: (c) 2013 Carpenter RL, et al. This is an open-access article distributed under the terms of the Creative Commons Attribution License, which permits unrestricted use, distribution, and reproduction in any medium, provided the original author and source are credited. 
Citation: Carpenter RL, Lo HW (2013) Regulation of Apoptosis by HER2 in Breast Cancer. J Carcinog Mutagen S7: 003. doi:10.4172/2157-2518. S7-003

ligands, such as FAS ligand binding to its receptor FAS. FAS is termed a death receptor and recruits adapter molecules, FAS-Associated Death Domain Protein (FADD) in the case of FAS. These adapter molecules dimerize and activate caspase 8 , another initiator caspase. There is some crossover between the extrinsic and intrinsic apoptotic pathways mediated by BH3-Interacting Domain Death Agonist (BID). After activation by death receptors, caspase 8 can cleave BID to form truncated BID (tBID), which localizes to the mitochondria and activates Bax/Bak-dependent MOMP leading to apoptosis. Both the extrinsic and intrinsic apoptotic pathways converge at activation of initiator caspases (e.g. caspase 8/9), which activate effector caspases (e.g. caspase 3), which have many targets in the cell that carry out the apoptosis program. These are brief descriptions of the mechanisms involved in the apoptotic program. To gain further insight to these mechanisms, the reader is referred to other reviews that describe these in more detail [6-8].

\section{HER2 Regulation of Apoptosis}

Overexpression of HER2 has been shown to suppress apoptosis through mechanisms that disrupt both the intrinsic and extrinsic apoptotic pathways. Maintenance of HER2 expression is also required for HER2-mediated suppression of apoptosis. Inhibition of Hsp90 in HER2-overexpressing cells resulted in degradation of HER2 protein and subsequent inactivation of downstream signaling including the PI3K-AKT pathway $[9,10]$. In addition to Hsp90, HER2 expression is also protected by Rab7, a GTPase that regulates endocytosis, as loss of Rab7 resulted in proteasome-mediated degradation of HER2
[11]. Thus, the proteins that maintain HER2 overexpression also help maintain suppression of apoptosis by HER2 signaling.

\section{HER2 regulation of the intrinsic pathway}

HER2 is a well-known activator of PI3K-AKT signaling and this pathway plays a large role in suppression of apoptosis by HER2 (Figure 1). AKT has been shown to directly phosphorylate the proapoptotic Bcl-2 protein Bad, which allows binding of 14-3-3 proteins that sequester Bad away from anti-apoptotic Bcl-2 proteins ultimately suppressing Bad-induced cell death [12]. AKT has also been shown to suppress Bim, another BH3-only pro-apoptotic protein [13]. AKT can phosphorylate several members of the forkhead family of transcription factors (FOXO), including FOXO3a, which leads to binding of 14-33 proteins to FOXO3a and sequestration from the nucleus $[14,15]$. Inhibition of FOXO3a transcriptional activity by nuclear export prevents FOXO3a-mediated expression of pro-apoptotic genes, including BIM [13-15]. Inhibition of FOXO family members by AKT also suppresses FOXO1-mediated suppression of the IAP survivin and prevents FOXO3a-mediated expression of the pro-apoptotic $\mathrm{BH} 3$ only protein Bnip3 [16,17]. In addition to regulation of these upstream participants in the intrinsic apoptotic pathway, AKT has been shown to directly phosphorylate caspase- 9 reducing its protease activity causing suppression of apoptosis [18].

HER2 also negatively regulates p53 function. HER2 suppresses p53 by two indirect mechanisms that are both mediated by AKT. First, it was observed that HER2 or AKT activation decreased expression and nuclear localization of p53 [19]. AKT was then found to directly

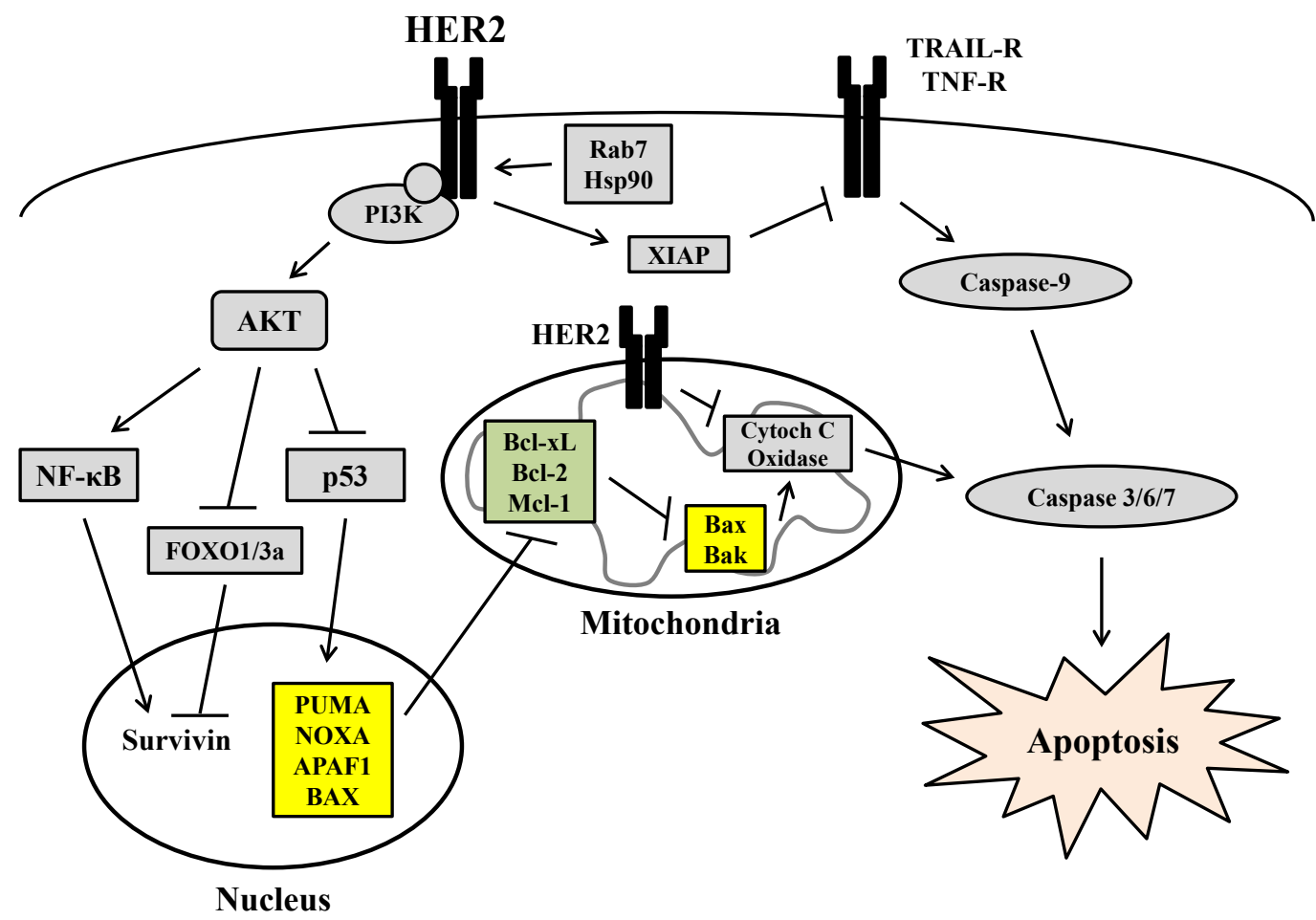

Figure 1: HER2 suppresses apoptosis via multiple mechanisms in both extrinsic and intrinsic apoptotic pathways. HER2 expression is maintained and stabilized by Rab7 and Hsp90. HER2 activates PI3K-AKT signaling, which directly suppresses FOXO1/3a through phosphorylation. Suppression of FOXO proteins relieves their repression of survivin. AKT also inhibits p53, which prevents p53-mediated expression of the pro-apoptotic genes PUMA, NOXA, APAF1, and BAX. HER2 activates NF-KB in an AKT-dependent manner, which increases expression of survivin. In addition, HER2 increases XIAP levels, which inhibits TRAIL-induced apoptosis. Lastly, HER2 localization to the mitochondria suppresses apoptosis by inhibition of cytochrome $c$ oxidase. Thus, HER2 initiates broad changes to cell signaling that results in cell survival and suppression of apoptosis in response to toxic therapies. 
phosphorylate MDM2 protein leading it into the nucleus where MDM2 can ubiquitinate p53 leading to its degradation [19]. The second mechanism involves AKT-mediated suppression of Alternate Reading Frame Protein (ARF). ARF interacts with and suppresses MDM2 from interacting with p53. HER2-overexpressing cells were observed to have reduced ARF expression in an AKT-dependent manner leading to increased MDM2 and reduced p53 [20]. Inhibition of p53 expression greatly inhibits apoptosis as p53 has been shown to upregulate several pro-apoptotic genes involved in the intrinsic apoptotic pathway including PUMA [21], NOXA [22], APAF-1 [23], and Bax [24]. In addition, p53 has been shown to suppress survivin [25] and Bcl-2 [24] expression. Thus, HER2 signaling enhances p53 degradation, preventing p53-mediated apoptosis.

Survivin appears to be important in HER2-mediated apoptosis suppression as it has a strong association with HER2 expression in patient tumors [26,27]. Knockdown of HER2 reduces survivin [28] and forced expression of HER2 increases survivin [29,30]. It was also observed that inhibition of the proteasome prevents loss of survivin protein with HER2 knockdown suggesting HER2 protects survivin from degradation [28]. As mentioned above, AKT-mediated down regulation of FOXO family members leads to increased survivin expression [16] and survivin expression can also be increased by AKT-mediated activation of NF- $\mathrm{BB}[31,32]$. Together, these results clearly show HER2 expression promotes survivin expression leads to decreased apoptosis.

Experimental manipulation of HER2 gives several clues to mechanisms of cell survival. Overexpression of HER 2 most commonly leads to upregulation of Bcl-2, Bcl-xL, Mcl-1, and survivin [30,33,34]. In accordance, there is a positive correlation between expression of HER2 with Mcl-1, Bcl-2, Bcl-xL, and survivin in patient tumors [26,27,34-37]. HER2 overexpression suppresses levels of miR15a and miR16, which target Bcl-2, leading to increased Bcl-2 expression [33]. Pharmacologic inhibition of $\mathrm{Bcl}-2$ sensitized HER2-overexpressing cells to lapatinib and knockdown of Bcl-2 increased responsiveness of HER2-overexpressing cells to tamoxifen [38,39]. These results suggest Bcl-2 is major target of HER2 in promoting cell survival. In addition to Bcl-2, knockdown of Mcl-1 and Bcl-xL sensitized HER2overexpressing cells to HER2-targeted therapy with observed increases in Bim expression [38,40,41]. Targeting HER2 with trastuzumab decreases expression of Bcl-2, Mcl-1, survivin, and phospho-Bad along with increased expression of the pro-apoptotic Bnip3 ultimately leading to cell death $[16,17,42-44]$. Treatment of HER2-overexpressing cells with lapatinib, the dual EGFR/HER2 inhibitor, increased expression of Bim, decreased expression of survivin and inhibited PI3K-mediated NF- $\kappa B$ activation [28,41, 45-47]. Overexpression and inhibition of HER2 indicate a dominant role of $\mathrm{Bcl}-2$ proteins in HER2-mediated protection from apoptosis.

Lastly, there is some recent evidence that HER2 can directly affect apoptosis. HER2 was recently detected in the mitochondria of HER2-overexpressing cells in a manner dependent on mitochondrial Hsp70 (mtHsp70) and active HER2 kinase activity [48]. One of the major functions of HER2 in the mitochondria was to reprogram cell metabolism toward glycolysis and away from oxidative phosphorylation [48]. However, investigators also observed that mitochondrial HER2 contributed to trastuzumab resistance by decreasing the activity of cytochrome $c$ oxidase in a HER2-dependent manner [48]. Furthermore, paclitaxel-induced apoptosis was reduced in cells expressing mitochondrial HER2 [48]. In addition, a HER2 fragment $(\sim 110 \mathrm{kDa})$ was identified as HER2 amino-terminal fragment
(H2NTF) that is expressed in approximately 60\% of HER2-positive breast cancer samples [49]. This fragment did not contain the kinase domain but did interact with the full-length HER2 receptor and prevented a trastuzumab-mediated decrease in cell viability [49]. Our lab observed that EGFR, a fellow ERBB RTK, could directly interact with the $\mathrm{BH} 3$-only protein PUMA, which prevents its localization to the mitochondria and promotion of apoptosis [50]. These results, suggest that ERBB family members take on a more direct role in suppressing apoptosis than previously thought.

\section{HER2 regulation of the extrinsic pathway}

While the regulation of the extrinsic apoptotic pathway by HER2 has been studied less, there is ample evidence HER2 also deregulates this pathway. Apoptosis in response to TNF- $\alpha$ was suppressed in HER2-overexpressing cells [51]. AKT-mediated activation of NF$\kappa B$ was required to suppress apoptosis with TNF- $\alpha$ exposure in these HER2-overexpressing cells [51]. Another study also observed that inhibition of PI3K-AKT signaling in HER2 overexpressing cells suppressed TRAIL-induced apoptosis [52]. TRAIL-induced apoptosis was enhanced by trastuzumab in these cells [52]. AKT signaling appears to be a general suppressor of TRAIL-induced apoptosis as active AKT has been seen to suppress apoptosis by TRAIL in multiple cancer types [53,54]. XIAP likely plays a significant role as knockdown of XIAP in HER2-overexpressing cells enhanced apoptosis by TRAIL and enhanced apoptosis with trastuzumab and lapatinib [55]. In addition to its effects on these pathways, HER2 mediates suppression of p53 as described above $[19,20]$. The death receptor Fas is a known target of p53 [56] and HER2-mediated suppression of p53 likely suppresses the cellular response to Fas-ligand.

\section{HER2 regulation of other factors that suppress apoptosis}

HER2 also regulates other proteins that appear to suppress apoptosis but are not directly involved in the intrinsic or extrinsic apoptotic cascade. The scaffolding protein p130Cas can bind to and enhance signaling of many HER2 downstream signaling molecules including FAK, PI3K, Crk, and Src among others [57]. Knockdown of of p130Cas in HER2-overexpressing cells induced apoptosis [58]. The mechanism by which apoptosis was induced was not elucidated but many of the pro-survival mechanisms of p130Cas involve signaling of its binding partners including PI3K, FAK, Src, and integrins among others [57]. Another factor involved is protein tyrosine kinase 6 (PTK6 or Brk), an intracellular tyrosine kinase that has no expression in normal breast tissue but robust expression in breast tumors [59]. Overexpression of HER2 induced PTK6 expression and knockdown of PTK6 in these cells induced apoptosis [60]. Previous reports suggest PTK6 regulates apoptosis by activating signaling pathways that enhance survival including AKT and STAT signaling [59]. Lastly, COX-2 may also contribute to HER2-mediated inhibition of apoptosis as HER2 activation upregulated COX-2 and inhibition of COX-2 induced apoptosis in HER2-overexpressing cells [61-63]. COX-2 has previously been shown to suppress apoptosis by PGE2-mediated activation of AKT and Ras signaling [64].

\section{Potential Drug Targets for HER2-positive Breast Cancer}

Apoptosis of HER2-overexpressing cells was shown to be enhanced by targeting HER2, PI3K-AKT, or Bcl-2 proteins in many pre-clinical studies. As such, most clinical attempts to suppress HER2overexpressed cancers have targeted HER2 or molecules downstream of HER2. The compounds Abt-737, Abt-263 (navitoclax), Abt-199, 
AT-101 and GX150-070 (obatoclax) are BH3 mimetics that target Bcl-2, Mcl-1, or Bcl-xL to suppress their anti-apoptotic function $[65,66]$. In clinical trials, these compounds have primarily been used in populations with liquid tumors or lung cancers leaving little clinical evidence of their utility in HER2-positive breast cancer. A preclinical study using a breast tumor xenograft model found that Abt-737 could lead to apoptosis in xenografts with the highest HER2 expression but overall tumor regression was very little [67]. However, the xenograft with the highest HER2 expression was much lower than tumors with HER2 amplification [67]. These BH3 mimetics have also been shown to be ineffective in cells without overexpression of anti-apoptotic $\mathrm{Bcl}-2$ proteins [68]. Considering Bcl-2, Mcl-1, and $\mathrm{Bcl}-\mathrm{xL}$ are upregulated in HER2-positive cancers, these proteins would seem to be attractive drug targets for HER2-positive cancers.

HER2 has been directly targeted by antibody approaches (e.g. trastuzumab) and kinase inhibitor approaches (e.g. lapatinib) that are often effective in patients combined with chemotherapy but patients also become resistant after long-term treatment [69]. Most recently, the antibody-drug conjugate T-DM1, which conjugates trastuzumab (T) with the cytotoxic agent mertansine (DM1), was FDA-approved for treating HER2-positive metastatic breast cancers with encouraging results in clinical trials in this population [70-72]. Another approach underway is using irreversible pan-ERBB inhibition (e.g. dacomitinib) with preclinical evidence of inhibition of HER2-overexpressing cells resistant to trastuzumab and lapatinib [73]

Considering patients will eventually become resistant to trastuzumab, an effective method of inhibiting HER2-positive tumor growth appears to be combination of HER2 inhibition along with targeting another downstream molecule. For instance, targeting HSP90 along with trastuzumab is more effective than either treatment alone in pre-clinical studies and this combination has shown antitumor activity in clinical trials [74-80]. The first of these clinical trials occurred in 2007 as a phase I dose-escalation study of trastuzumab $(4 \mathrm{mg} / \mathrm{kg})$ along with tanespimycin (or 17-AAG) doses of 225, 300, 375, and $450 \mathrm{mg} / \mathrm{m}^{2}$ on 25 patients with advanced solid tumors with 14 of the patients having previous trastuzumab treatment [77]. The dose limiting toxicity of tanespimycin was $375 \mathrm{mg} / \mathrm{m}^{2}$ with mild to moderate toxicity symptoms of fatigue, nausea, diarrhea, emesis, headache, and rash [77]. Antitumor activity was assessed by RECIST criteria, which resulted in 1 partial response while another 4 patients showed tumor regression, with regression as high as $70 \%$, and all had HER2-positive metastatic breast cancer and previous trastuzumab treatment [77]. An additional 3 patients had stable disease and two were confirmed to be HER2positive with 1 having previous trastuzumab treatment [77]. A more recent phase I trial was conducted using trastuzumab $(4 \mathrm{mg} / \mathrm{kg})$ along with alvespimycin (17-DMAG) doses of 60,80 , and $100 \mathrm{mg} / \mathrm{m}^{2}$ on 28 patients with advanced solid tumors with 24 of these patients having previous trastuzumab treatment [74]. Dose limiting toxicity was $80 \mathrm{mg} / \mathrm{m}^{2}$ with common mild to moderate toxicities being diarrhea, fatigue, myalgia, arthralgia, nausea, blurry vision, headache, back pain, and dry eyes [74]. One patient showed a partial response and 7 patients displayed stable disease and all had HER2-positive metastatic breast cancer and previous trastuzumab treatment. This resulted in a clinical benefit rate of $29 \%$ ( $\mathrm{CR}+\mathrm{PR}+\mathrm{SD}>6$ months) in evaluable patients [74].

The most recent trial for HSP90 inhibitors is a phase 2 trial on patients with advanced or metastatic HER2-positive breast cancer and prior trastuzumab treatment that were treated with $300 \mathrm{mg} / \mathrm{m}^{2}$ retaspimycin $\mathrm{HCl}$ (IPI-504) combined with $6 \mathrm{mg} / \mathrm{kg}$ trastuzumab every three weeks [76]. Twenty-six patients were enrolled and received a median of three treatment cycles. The best response observed was stable disease in 16 patients but was not enough to warrant trial expansion. However, toxicity symptoms were less than expected leading the authors to suggest a similar trial with higher retaspimycin HCL dosage in hopes of obtaining a greater response rate [76]. Similar to HSP90 inhibitors, pre-clinical studies show that inhibition of PI3K with trastuzumab is greater than either treatment alone in HER2overexpressing cells $[16,81,82]$. There are several phase I/II clinical trials currently ongoing or recently completed (but without publication of results) to assess safety and efficacy of targeting HER2 and PI3K. These trials are using PI3K inhibitors such as GDC-0941, BKM120, BEZ235, or XL147 and targeting HER2 with trastuzumab or lapatinib. Considering the large role PI3K plays in transmitting HER2 signaling throughout the cell, results of these trials will provide great insight to the treatment of HER2-positive breast cancer. Pre-clinical and clinical results seem to point to the need for targeting multiple components of HER2-mediated cell survival mechanisms in hopes of achieving stable, long-term inhibition of HER2-overexpressing tumor growth.

\section{Conclusions}

The HER2 signaling network spans across many functions of the cell and influences cell behavior greatly, especially in breast cancer cells overexpressing HER2. As such, it is not surprising to find that breast cancer cells dependent on HER2 have utilized multiple mechanisms to suppress apoptosis for survival. Evidence suggests that HER2 can inhibit both the intrinsic and extrinsic apoptotic pathways (Figure 1). Future studies need to elucidate mechanisms by which therapyresistant HER2-overexpressing cells are able to suppress apoptosis to further aid in designing effective clinical treatment regimens. In addition, the relevance of HER2 localization to the mitochondria should be investigated in the context of apoptosis as well as the ability of HER2 to directly influence apoptotic regulators. Future drug development for HER2-overexpressed cancers should target some of these well-known mechanisms by which HER2 suppresses apoptosis and find combinations of targets that are most effective to limit clinical side effects. Breast cancer death rates continue to fall [83] and future clinical and pre-clinical study targeting HER2 will only in aid this progression.

\section{Acknowledgements}

This study was supported by the NIH grant K01-CA118423, and W81XWH-11-1-0600 from the U.S. Department of Defense, the Beez Foundation, and the Intramural Division of Surgical Sciences Dani P. Bolognesi, Ph.D. Award and Clarence Gardner, Ph.D. Award (to H.-W. L).

\section{Reference}

1. Slamon DJ, Clark GM, Wong SG, Levin WJ, Ullrich A, et al. (1987) Human breast cancer: correlation of relapse and survival with amplification of the HER 2/neu oncogene. Science 235: 177-182

2. Slamon DJ, Godolphin W, Jones LA, Holt JA, Wong SG, et al. (1989) Studies of the HER-2/neu proto-oncogene in human breast and ovarian cancer. Science 244: 707-712.

3. Yarden Y, Sliwkowski MX (2001) Untangling the ErbB signalling network. Nat Rev Mol Cell Biol 2: 127-137.

4. Hanahan D, Weinberg RA (2011) Hallmarks of cancer: the next generation Cell 144: 646-674.

5. Susnow N, Zeng L, Margineantu D, Hockenbery DM (2009) Bcl-2 family proteins as regulators of oxidative stress. Semin Cancer Biol 19: 42-49.

6. Galluzzi L, Kepp O, Kroemer G (2012) Mitochondria: master regulators of danger signalling. Nat Rev Mol Cell Biol 13: 780-788.

7. Tait SW, Green DR (2010) Mitochondria and cell death: outer membrane permeabilization and beyond. Nat Rev Mol Cell Biol 11: 621-632. 
8. Youle RJ, Strasser A (2008) The BCL-2 protein family: opposing activities that mediate cell death. Nat Rev Mol Cell Biol 9: 47-59.

9. Solit DB, Basso AD, Olshen AB, Scher HI, Rosen N (2003) Inhibition of heat shock protein 90 function down-regulates Akt kinase and sensitizes tumors to Taxol. Cancer Res 63: 2139-2144.

10. Yan YY, Zheng LS, Zhang X, Chen LK, Singh S, et al. (2011) Blockade of Her2/neu Binding to Hsp90 by Emodin Azide Methyl Anthraquinone Derivative Induces Proteasomal Degradation of Her2/neu. Molecular Pharmaceutics 8 : 1687-1697.

11. Wang T, Zhang M, Ma Z, Guo K, Tergaonkar V, et al. (2012) A role of Rab7 in stabilizing EGFR-Her2 and in sustaining Akt survival signal. J Cell Physiol 227: $2788-2797$

12. Datta SR, Dudek H, Tao X, Masters S, Fu H, et al. (1997) Akt phosphorylation of BAD couples survival signals to the cell-intrinsic death machinery. Cell 91 : 231-241.

13. Dijkers PF, Medema RH, Pals C, Banerii L, Thomas NS, et al. (2000) Forkhead transcription factor FKHR-L1 modulates cytokine-dependent transcriptional regulation of p27(KIP1). Mol Cell Biol 20: 9138-9148.

14. Brunet A, Bonni A, Zigmond MJ, Lin MZ, Juo P, et al. (1999) Akt promotes cell survival by phosphorylating and inhibiting a Forkhead transcription factor. Cell 96: 857-868.

15. Dijkers PF, Birkenkamp KU, Lam EW, Thomas NS, Lammers JW, et al (2002) FKHR-L1 can act as a critical effector of cell death induced by cytokine withdrawal: protein kinase B-enhanced cell survival through maintenance of mitochondrial integrity. J Cell Biol 156: 531-542.

16. Chakrabarty A, Bhola NE, Sutton C, Ghosh R, Kuba MG, et al. (2013) Trastuzumab-resistant cells rely on a HER2-PI3K-FoxO-survivin axis and are sensitive to PI3K inhibitors. Cancer Res 73: 1190-1200.

17. Real PJ, Benito A, Cuevas J, Berciano MT, de Juan A, et al. (2005) Blockade of epidermal growth factor receptors chemosensitizes breast cancer cells through up-regulation of Bnip3L. Cancer Res 65: 8151-8157.

18. Cardone MH, Roy N, Stennicke HR, Salvesen GS, Franke TF, et al. (1998) Regulation of cell death protease caspase- 9 by phosphorylation. Science 282 : 1318-1321.

19. Zhou BP, Liao Y, Xia W, Zou Y, Spohn B, et al. (2001) HER-2/neu induces p53 ubiquitination via Akt-mediated MDM2 phosphorylation. Nat Cell Biol 3: 973-982.

20. Zhang Y, Yang HY, Zhang XC, Yang H, Tsai M, et al. (2004) Tumor suppressor ARF inhibits HER-2/neu-mediated oncogenic growth. Oncogene 23: 71327143.

21. Han J, Flemington C, Houghton AB, Gu Z, Zambetti GP, et al. (2001) Expression of bbc3, a pro-apoptotic $\mathrm{BH} 3$-only gene, is regulated by diverse cell death and survival signals. Proc Natl Acad Sci U S A 98: 11318-11323.

22. Oda E, Ohki R, Murasawa H, Nemoto J, Shibue T, et al. (2000) Noxa, a $\mathrm{BH} 3-$ only member of the $\mathrm{Bcl}-2$ family and candidate mediator of p53-induced apoptosis. Science 288: 1053-1058.

23. Moroni MC, Hickman ES, Lazzerini Denchi E, Caprara G, Colli E, et al. (2001) Apaf-1 is a transcriptional target for E2F and p53. Nat Cell Biol 3: 552-558.

24. Selvakumaran M, Lin HK, Miyashita T, Wang HG, Krajewski S, et al. (1994) Immediate early up-regulation of bax expression by p53 but not TGF beta 1: a paradigm for distinct apoptotic pathways. Oncogene 9: 1791-1798.

25. Sah NK, Khan Z, Khan GJ, Bisen PS (2006) Structural, functional and therapeutic biology of survivin. Cancer Lett 244: 164-171.

26. Ryan BM, Konecny GE, Kahlert S, Wang HJ, Untch M, et al. (2006) Survivin expression in breast cancer predicts clinical outcome and is associated with HER2, VEGF, urokinase plasminogen activator and PAI-1. Ann Oncol 17: 597 604

27. Singh M, Bleile MJ, Shroyer AL, Heinz D, Jarboe EA, et al. (2004) Analysis of survivin expression in a spectrum of benign to malignant lesions of the breast. Applied immunohistochemistry \& molecular morphology : AIMM / official publication of the Society for Applied Immunohistochemistry 12: 296-304.

28. Xia W, Bisi J, Strum J, Liu L, Carrick K, et al. (2006) Regulation of survivin by ErbB2 signaling: therapeutic implications for ErbB2-overexpressing breast cancers. Cancer Res 66: 1640-1647.
29. Asanuma H, Torigoe T, Kamiguchi K, Hirohashi Y, Ohmura T, et al. (2005) Survivin expression is regulated by coexpression of human epidermal growth factor receptor 2 and epidermal growth factor receptor via phosphatidylinositol 3-kinase/AKT signaling pathway in breast cancer cells. Cancer Res 65: 1101811025.

30. Siddiqa A, Long LM, Li L, Marciniak RA, Kazhdan I (2008) Expression of HER 2 in MCF-7 breast cancer cells modulates anti-apoptotic proteins Survivin and $\mathrm{Bcl}-2$ via the extracellular signal-related kinase (ERK) and phosphoinositide-3 kinase (PI3K) signalling pathways. BMC Cancer 8: 129

31. Papanikolaou V, Iliopoulos D, Dimou I, Dubos S, Kappas C, et al. (2011) Survivin regulation by HER2 through NF-10 ${ }^{\circ} \mathrm{B}$ and c-myc in irradiated breast cancer cells. J Cell Mol Med 15: 1542-1550.

32. Zhao P, Meng Q, Liu LZ, You YP, Liu N, et al. (2010) Regulation of survivin by PI3K/Akt/p70S6K1 pathway. Biochem Biophys Res Commun 395: 219-224

33. Cittelly DM, Das PM, Salvo VA, Fonseca JP, Burow ME, et al. (2010) Oncogenic HER2\{Delta\}16 suppresses miR-15a/16 and deregulates BCL-2 to promote endocrine resistance of breast tumors. Carcinogenesis 31: 2049-2057.

34. Kumar R, Mandal M, Lipton A, Harvey H, Thompson CB (1996) Overexpression of HER2 modulates bcl-2, bcl-XL, and tamoxifen-induced apoptosis in human MCF-7 breast cancer cells. Clin Cancer Res 2: 1215-1219.

35. Henson ES, Hu X, Gibson SB (2006) Herceptin sensitizes ErbB2 overexpressing cells to apoptosis by reducing antiapoptotic $\mathrm{Mcl}-1$ expression Clin Cancer Res 12: 845-853.

36. Rexhepaj E, Jirstrom K, O'Connor DP, O'Brien SL, Landberg G, et al. (2010) Validation of cytoplasmic-to-nuclear ratio of survivin as an indicator of improved prognosis in breast cancer. BMC Cancer 10: 639.

37. Siziopikou KP, Khan S (2005) Correlation of HER2 gene amplification with expression of the apoptosis-suppressing genes bcl-2 and bcl-x-L in ductal carcinoma in situ of the breast. Applied immunohistochemistry \& molecular morphology : AIMM / official publication of the Society for Applied Immunohistochemistry 13: 14-18.

38. Cruickshanks N, Hamed HA, Bareford MD, Poklepovic A, Fisher PB, et al (2012) Lapatinib and obatoclax kill tumor cells through blockade of ERBB1/3/4 and through inhibition of BCL-XL and MCL-1. Mol Pharmacol 81: 748-758.

39. Martin AP, Mitchell C, Rahmani M, Nephew KP, Grant S, et al. (2009) Inhibition of MCL-1 enhances lapatinib toxicity and overcomes lapatinib resistance via BAK-dependent autophagy. Cancer Biol Ther 8: 2084-2096.

40. Campone M, Noël B, Couriaud C, Grau M, Guillemin Y, et al. (2011) c-Myc dependent expression of pro-apoptotic Bim renders HER2-overexpressing breast cancer cells dependent on anti-apoptotic Mcl-1. Mol Cancer 10: 110.

41. Mitchell C, Yacoub A, Hossein H, Martin AP, Bareford MD, et al. (2010) Inhibition of MCL-1 in breast cancer cells promotes cell death in vitro and in vivo. Cancer Biol Ther 10: 903-917.

42. Oliveras-Ferraros C, Vazquez-Martin A, Cufí S, Torres-Garcia VZ, Sauri-Nada T, et al. (2011) Inhibitor of Apoptosis (IAP) survivin is indispensable for survival of HER2 gene-amplified breast cancer cells with primary resistance to HER1/2targeted therapies. Biochemical and Biophysical Research Communications 407: 412-419.

43. Real PJ, Cao Y, Wang R, Nikolovska-Coleska Z, Sanz-Ortiz J, et al. (2004) Breast cancer cells can evade apoptosis-mediated selective killing by a nove small molecule inhibitor of Bcl-2. Cancer Res 64: 7947-7953.

44. Seoane S, Montero JC, Ocaña A, Pandiella A (2010) Effect of multikinase inhibitors on caspase-independent cell death and DNA damage in HER2 overexpressing breast cancer cells. J Natl Cancer Inst 102: 1432-1446.

45. Faber AC, Corcoran RB, Ebi H, Sequist LV, Waltman BA, et al. (2011) BIM expression in treatment-naive cancers predicts responsiveness to kinase inhibitors. Cancer Discov 1: 352-365.

46. Ma C, Zuo W, Wang X, Wei L, Guo Q, et al. (2013) Lapatinib inhibits the activation of $\mathrm{NF}-\hat{I}^{\circ} \mathrm{B}$ through reducing phosphorylation of Îे $^{\circ} \mathrm{B}-\hat{\mathrm{I}} \pm$ in breas cancer cells. Oncol Rep 29: 812-818.

47. Tanizaki J, Okamoto I, Fumita S, Okamoto W, Nishio K, et al. (2011) Roles of BIM induction and survivin downregulation in lapatinib-induced apoptosis in breast cancer cells with HER2 amplification. Oncogene 30: 4097-4106.

48. Ding Y, Liu Z, Desai S, Zhao Y, Liu H, et al. (2012) Receptor tyrosine kinase ErbB2 translocates into mitochondria and regulates cellular metabolism. Nat Commun 3: 1271. 
Citation: Carpenter RL, Lo HW (2013) Regulation of Apoptosis by HER2 in Breast Cancer. J Carcinog Mutagen S7: 003. doi:10.4172/2157-2518. S7-003

49. Morancho B, Parra-Palau JL, Ibrahim YH, Bernadó Morales C, Peg V, et al. (2013) A dominant-negative N-terminal fragment of HER2 frequently expressed in breast cancers. Oncogene 32: 1452-1459.

50. Zhu H, Cao X, Ali-Osman F, Keir S, Lo HW (2010) EGFR and EGFRvIII interact with PUMA to inhibit mitochondrial translocalization of PUMA and PUMAmediated apoptosis independent of EGFR kinase activity. Cancer Lett 294 101-110.

51. Zhou BP, Hu MC, Miller SA, Yu Z, Xia W, et al. (2000) HER-2/neu blocks tumor necrosis factor-induced apoptosis via the Akt/NF-kappaB pathway. J Biol Chem 275: 8027-8031.

52. Cuello M, Ettenberg SA, Clark AS, Keane MM, Posner RH, et al. (2001) DownRegulation of the erbB-2 Receptor by Trastuzumab (Herceptin) Enhances Tumor Necrosis Factor-related Apoptosis-inducing Ligand-mediated Apoptosis in Breast and Ovarian Cancer Cell Lines that Overexpress erbB-2. Cancer Research 61: 4892-4900.

53. Kandasamy K, Srivastava RK (2002) Role of the phosphatidylinositol 3'-kinase/ PTEN/Akt kinase pathway in tumor necrosis factor-related apoptosis-inducing ligand-induced apoptosis in non-small cell lung cancer cells. Cancer Res 62 : 4929-4937.

54. Thakkar H, Chen X, Tyan F, Gim S, Robinson H, et al. (2001) Pro-survival function of Akt/protein kinase $B$ in prostate cancer cells. Relationship with TRAIL resistance. J Biol Chem 276: 38361-38369.

55. Foster FM, Owens TW, Tanianis-Hughes J, Clarke RB, Brennan K, et al. (2009) Targeting inhibitor of apoptosis proteins in combination with ErbB antagonists in breast cancer. Breast Cancer Res 11: R41.

56. Owen-Schaub LB, Zhang W, Cusack JC, Angelo LS, Santee SM, et al. (1995) Wild-type human p53 and a temperature-sensitive mutant induce Fas/APO-1 expression. Mol Cell Biol 15: 3032-3040.

57. Defilippi P, Di Stefano P, Cabodi S (2006) p130Cas: a versatile scaffold in signaling networks. Trends Cell Biol 16: 257-263.

58. Cabodi S, Tinnirello A, Di Stefano P, Bisarò B, Ambrosino E, et al. (2006) p130Cas as a new regulator of mammary epithelial cell proliferation, survival, and HER2-neu oncogene-dependent breast tumorigenesis. Cancer Res 66 : $4672-4680$.

59. Brauer PM, Tyner AL (2010) Building a better understanding of the intracellular tyrosine kinase PTK6 - BRK by BRK. Biochim Biophys Acta 1806: 66-73.

60. Ai M, Liang K, Lu Y, Qiu S, Fan Z (2013) Brk/PTK6 cooperates with HER2 and $\mathrm{Src}$ in regulating breast cancer cell survival and epithelial-to-mesenchymal transition. Cancer Biol Ther 14: 237-245

61. Chen B, Su B, Chen S (2009) A COX-2 inhibitor nimesulide analog selectively induces apoptosis in Her2 overexpressing breast cancer cells via cytochrome $c$ dependent mechanisms. Biochem Pharmacol 77: 1787-1794.

62. Simeone AM, Li YJ, Broemeling LD, Johnson MM, Tuna M, et al. (2004) Cyclooxygenase-2 is essential for HER2/neu to suppress $\mathrm{N}$ - (4-hydroxyphenyl) retinamide apoptotic effects in breast cancer cells. Cancer Res 64: 1224-1228.

63. Vadlamudi R, Mandal M, Adam L, Steinbach G, Mendelsohn J, et al. (1999) Regulation of cyclooxygenase-2 pathway by HER2 receptor. Oncogene 18 : 305-314.

64. Ghosh N, Chaki R, Mandal V, Mandal SC (2010) COX-2 as a target for cancer chemotherapy. Pharmacol Rep 62: 233-244.

65. Khaw SL, Huang DC, Roberts AW (2011) Overcoming blocks in apoptosis with BH3-mimetic therapy in haematological malignancies. Pathology 43: 525-535.

66. Souers AJ, Leverson JD, Boghaert ER, Ackler SL, Catron ND, et al. (2013) ABT-199, a potent and selective BCL-2 inhibitor, achieves antitumor activity while sparing platelets. Nat Med 19: 202-208.

67. Kutuk O, Letai A (2008) Alteration of the mitochondrial apoptotic pathway is key to acquired paclitaxel resistance and can be reversed by ABT-737. Cancer Res 68: 7985-7994.
68. Oakes SR, Vaillant F, Lim E, Lee L, Breslin K, et al. (2012) Sensitization of $\mathrm{BCL}-2$-expressing breast tumors to chemotherapy by the $\mathrm{BH} 3$ mimetic ABT737. Proc Natl Acad Sci U S A 109: 2766-2771.

69. Park JW, Neve RM, Szollosi J, Benz CC (2008) Unraveling the biologic and clinical complexities of HER2. Clin Breast Cancer 8: 392-401.

70. Krop IE, Beeram M, Modi S, Jones SF, Holden SN, et al. (2010) Phase I study of trastuzumab-DM1, an HER2 antibody-drug conjugate, given every 3 weeks to patients with HER2-positive metastatic breast cancer. J Clin Oncol 28: 26982704.

71. Krop IE, LoRusso P, Miller KD, Modi S, Yardley D, et al. (2012) A phase II study of trastuzumab emtansine in patients with human epidermal growth factor receptor 2-positive metastatic breast cancer who were previously treated with trastuzumab, lapatinib, an anthracycline, a taxane, and capecitabine. Journal of clinical oncology : official journal of the American Society of Clinical Oncology 30: 3234-3241.

72. Verma S, Miles D, Gianni L, Krop IE, Welslau M, et al. (2012) Trastuzumab emtansine for HER2-positive advanced breast cancer. N Engl J Med 367 : 1783-1791.

73. Kalous O, Conklin D, Desai AJ, O'Brien NA, Ginther C, et al. (2012) Dacomitinib (PF-00299804), an irreversible Pan-HER inhibitor, inhibits proliferation of HER2-amplified breast cancer cell lines resistant to trastuzumab and lapatinib. Mol Cancer Ther 11: 1978-1987.

74. Jhaveri K, Miller K, Rosen L, Schneider B, Chap L, et al. (2012) A phase I doseescalation trial of trastuzumab and alvespimycin hydrochloride (KOS-1022; 17 DMAG) in the treatment of advanced solid tumors. Clinical cancer research an official journal of the American Association for Cancer Research 18: 5090 5098.

75. Leow CC, Chesebrough J, Coffman KT, Fazenbaker CA, Gooya J, et al. (2009) Antitumor efficacy of IPI-504, a selective heat shock protein 90 inhibitor against human epidermal growth factor receptor 2-positive human xenograft models as a single agent and in combination with trastuzumab or lapatinib. Mol Cancer Ther 8: 2131-2141.

76. Modi S, Saura C, Henderson C, Lin NU, Mahtani R, et al. (2013) A multicenter trial evaluating retaspimycin $\mathrm{HCL}$ (IPI-504) plus trastuzumab in patients with advanced or metastatic HER2-positive breast cancer. Breast Cancer Res Trea 139: 107-113.

77. Modi S, Stopeck AT, Gordon MS, Mendelson D, Solit DB, et al. (2007) Combination of trastuzumab and tanespimycin (17-AAG, KOS-953) is safe and active in trastuzumab-refractory HER-2 overexpressing breast cancer: a phase I dose-escalation study. J Clin Oncol 25: 5410-5417.

78. Ono N Yamazaki T, Nakanishi Y, Fujii T, Sakata K, et al (2012) Preclinica antitumor activity of the novel heat shock protein 90 inhibitor $\mathrm{CH} 5164840$ against human epidermal growth factor receptor 2 (HER2)-overexpressing cancers. Cancer Sci 103: 342-349.

79. Wainberg ZA, Anghel A, Rogers AM, Desai AJ, Kalous O, et al. (2013) Inhibition of HSP90 with AUY922 induces synergy in HER2-amplified trastuzumabresistant breast and gastric cancer. Mol Cancer Ther 12: 509-519.

80. Zsebik B, Citri A, Isola J, Yarden Y, Szöllosi J, et al. (2006) Hsp90 inhibito 17-AAG reduces ErbB2 levels and inhibits proliferation of the trastuzumab resistant breast tumor cell line JIMT-1. Immunol Lett 104: 146-155.

81. Junttila TT, Akita RW, Parsons K, Fields C, Lewis Phillips GD, et al. (2009) Ligand-independent HER2/HER3/PI3K complex is disrupted by trastuzumab and is effectively inhibited by the PI3K inhibitor GDC-0941. Cancer Cell 15: 429-440.

82. Yao E, Zhou W, Lee-Hoeflich ST, Truong T, Haverty PM, et al. (2009) Suppression of HER2/HER3-mediated growth of breast cancer cells with combinations of GDC-0941 PI3K inhibitor, trastuzumab, and pertuzumab. Clin Cancer Res 15: 4147-4156.

83. ACS (2013) Cancer Facts \& Figures 2013. American Cancer Society, Atlanta GA
This article was originally published in a special issue, Signal transductionCancer handled by Editor(s). Dr. Li-Mei Chen, University of Central Florida College of Medicine, United States 